\title{
Strong Spin-Orbit Splitting on Bi Surfaces
}

\author{
Yu. M. Koroteev, ${ }^{1,2}$ G. Bihlmayer, ${ }^{3}$ J. E. Gayone, ${ }^{4,5}$ E. V. Chulkov, ${ }^{1,6}$ S. Blügel, ${ }^{3}$ P. M. Echenique,,${ }^{1,6}$ and Ph. Hofmann ${ }^{4, *}$ \\ ${ }^{1}$ Donostia International Physics Center (DIPC), 20018 San Sebastián/Donostia, Basque Country, Spain \\ ${ }^{2}$ Institute of Strength Physics and Materials Science, Russian Academy of Sciences, 634021, Tomsk, Russia \\ ${ }^{3}$ Institut für Festkörperforschung, Forschungszentrum Jülich, D-52425 Jülich, Germany \\ ${ }^{4}$ Institute for Storage Ring Facilities, University of Aarhus, DK-8000 Aarhus C, Denmark \\ ${ }^{5}$ Centro Atomico Bariloche and CONICET, 8400 S.C. de Bariloche, Argentina \\ ${ }^{6}$ Departamento de Física de Materiales and Centro Mixto CSIC-UPV/EHU, Facultad de Ciencias Químicas, UPV/EHU, \\ Apartado 1072, 20080 San Sebastián, Basque Country, Spain
}

(Received 25 February 2004; published 23 July 2004)

\begin{abstract}
Using first-principles calculations and angle-resolved photoemission, we show that the spin-orbit interaction leads to a strong splitting of the surface-state bands on low-index surfaces of $\mathrm{Bi}$. The dispersion of the states and the corresponding Fermi surfaces are profoundly modified in the whole surface Brillouin zone. We discuss the implications of these findings with respect to a proposed surface charge density wave on $\mathrm{Bi}(111)$ as well as to the surface screening, surface spin-density waves, electron (hole) dynamics in surface states, and to possible applications to the spintronics.
\end{abstract}

DOI: 10.1103/PhysRevLett.93.046403

PACS numbers: 71.18.+y, 73.20.-r, 73.25.+i, 79.60.-i

Recently, spin-orbit coupling (SOC) on surfaces and the resulting splitting of the surface-state bands has attracted considerable attention. While it is a wellestablished fact that the reduction of coordination at surfaces and in thin films can lead to pronounced magnetic effects, the discovery of a small splitting in the band of the $s p$ surface state on the nonmagnetic Au(111) surface and its interpretation as being due to SOC by LaShell et al. [1] came as a surprise. More sophisticated angleresolved photoemission (ARPES) investigations and calculations have meanwhile confirmed this splitting [2] and the combination of the experimental results with firstprinciples calculations do indeed prove that the SOC is causing it [2,3]. Later, larger SOC-induced splittings were found on the Li-covered surfaces of $\mathrm{W}$ and Mo [4] and the predicted difference in spin orientations for $\mathrm{H}$ on $\mathrm{W}(110)$ was confirmed experimentally using spinresolved ARPES [5]. Since these surface states contribute only very little to the density of states at the Fermi level, the observed spin-orbit or Rashba splitting of these states will not show up in transport phenomena. On the other hand, surface states of a semimetal would give a prominent contribution [6,7], which could make these systems interesting for applications in the field of spintronics. The surfaces of the semimetal Bi seem to be ideal to advance our understanding of SOC on surfaces and how it manifests itself in experiments. Of particular interest are the influence of the SOC on the electron-phonon coupling [8], electron and hole dynamics [9], and the possible formation of surface charge (spin) density waves. The occurrence of strong SOC in low-dimensional structures of nonmagnetic materials could also have applications such as spin-filter devices.

ARPES measurements of the Fermi surface (FS) and surface states were recently performed by Ast and Höchst for $\mathrm{Bi}(111)$ [10]. They interpreted the obtained FS in terms of two different surface bands which are not degenerate at the $\bar{\Gamma}$ point. Based on this electronic structure, they proposed a possible mechanism for the formation of surface charge density waves (CDWs) on $\mathrm{Bi}(111)$ [11]. Agergaard et al. [7] measured surface states and the FS on $\mathrm{Bi}(110)$. They pointed out that these surface states should be completely nondegenerate because of spin-orbit splitting but, as we show below, the splitting is so large that an easy identification of the spin-split bands was not possible.

Bulk $\mathrm{Bi}$ is a semimetal where the strong spin-orbit interaction essentially accounts for the existence of the hole FS at the $T$ point [12] but does not lead to any lifting of the spin degeneracy because of inversion symmetry. However, the high atomic number of $\mathrm{Bi}(83)$ and the pronounced splitting in the atomic $p$ levels [the atomic $p_{3 / 2}-p_{1 / 2}$ splitting in $\mathrm{Bi}(1.5 \mathrm{eV})$ is 3 times stronger than in $\mathrm{Au}(0.47 \mathrm{eV})$ [13]] should lead to an observable splitting of the surface-state bands.

In this Letter, we show that indeed the surface states on low-index surfaces of Bi exhibit a spin-orbit splitting of the bands which is by far stronger than any case reported so far. We prove this by combining the results of firstprinciples calculations with high-resolution measurements of the electronic structure by ARPES. The results of the calculation agree well with experiment but only if the SOC and hence the removal of the spin degeneracy are taken into account. We find that the SOC-induced splitting is an essential ingredient for the description of the electronic structure: it profoundly changes the surface-state dispersion and the corresponding Fermi surfaces on all the Bi surfaces of interest. In particular, it is responsible for the existence of the six FS hole lobes in the $\bar{\Gamma} \bar{M}$ symmetry directions for $\mathrm{Bi}(111)$, and it also leads to 
correct dimensions of the electron FS hexagon around the $\bar{\Gamma}$ point. Our relativistic calculation demonstrates that, despite the existence of nesting at the electron FS hexagon of $\mathrm{Bi}(111)$, the formation of a CDW [11] appears to be improbable since this nesting couples the states with the same energy but different spin, $\epsilon(\mathbf{k}, \uparrow)=\epsilon(-\mathbf{k}, \downarrow)$. It could lead rather to the formation of a spin-density wave and not to a CDW.

Here we present results for three surfaces: $\operatorname{Bi}(111)$, $\operatorname{Bi}(110)$, and $\operatorname{Bi}(100)$. The calculations have been performed by using the full-potential linearized augmented plane-wave method in film geometry [14] as implemented in the FLEUR program and local density approximation for the description of exchange-correlation potential. SOC is included self-consistently as described in Ref. [15]. All the Bi surfaces were simulated by a 22 layer film embedded in vacuum. One side of the film was terminated with hydrogen to avoid interaction between the surface states of the two surfaces of the film. The $\mathrm{H}$ atoms were placed a distance of $\approx 2 \AA$ from the Bi. On the other side of the film, the termination was chosen such that the interlayer distance between surface and subsurface atoms was the shorter of two possible terminations. For the calculations a plane-wave cutoff of $K_{\max }=$ 3.4 (a.u. $)^{-1}$ was used and the surface Brillouin zone (SBZ) was sampled with up to $121 \mathbf{k}_{\|}$points.

We also show surface states measured with angleresolved photoemission spectroscopy. The experiments were performed at the SGM-3 beam line of the synchrotron radiation source ASTRID in Aarhus [16]. All surfaces were prepared from mechanically polished single-crystal surfaces, which were cleaned in situ by cycles of $\mathrm{Ne}$ ion bombardment and annealing to about $473 \mathrm{~K}$. This resulted in well-ordered and clean surfaces as judged by low energy electron diffraction and Auger electron spectroscopy, respectively. The total energy resolution for the data shown below is better than $35 \mathrm{meV}$. The angular resolution of the analyzer is $\pm 0.5^{\circ}$. The samples were cooled to approximately $30 \mathrm{~K}$.

Before discussing our results, we briefly explain the symmetries relevant for the spin-orbit splitting of electronic bands. Time-reversal symmetry requires that $\epsilon(\mathbf{k}, \uparrow)=\epsilon(-\mathbf{k}, \downarrow)$. This means that if one has a surface state at $\mathbf{k}$ with a binding energy $\epsilon$ and a spin $\uparrow$, then there must also be a state at $\mathbf{- k}$ with the same energy but spin $\downarrow$. This has to be combined with the usual space group symmetry. The combination has two consequences. First, if the space group contains inversion symmetry $[\epsilon(\mathbf{k}, \uparrow)=$ $\epsilon(-\mathbf{k}, \uparrow)]$, the bands are obviously doubly degenerate. This can happen in the bulk but not at the surface. Second, in the case of surface states, the splitting has to be zero at some special points of the surface Brillouin zone. The application of time-reversal symmetry alone yields that one of these points is the center of the zone $\bar{\Gamma}$ for which $\mathbf{k}_{\|}=0$. This is indeed observed in the dispersion of the spin-orbit split states on $\mathrm{Au}(111)$ [1]. The combination of time-reversal symmetry with translational symmetry dictates that the splitting must also be zero for any point which is situated halfway between two $\bar{\Gamma}$ points.

Figure 1 shows the electronic structure of $\mathrm{Bi}(111)$ together with the projected bulk band structure for the (111) surface calculated with and without SOC. The projection was calculated using the tight-binding model of Ref. [17]. For clarity, we show only the surface states, which are located on the clean surface of the slab. In the case without SOC, we find a parabolic $\bar{\Gamma}$ surface state located in the nonrelativistic energy gap. Around $\bar{\Gamma}$ this surface-state band gives an electron FS hexagon. When the SOC term is included, it results in a spin splitting of the surface state in all the symmetry directions and leaves it degenerate only at $\bar{\Gamma}$ and at $\bar{M}$. The latter is expected because $\bar{M}$ is a high-symmetry point on the SBZ boundary which lies, in contrast to, e.g., $\bar{K}$, in the middle between two $\bar{\Gamma}$ points. Around $\bar{\Gamma}$ this relativistic surface state is degenerate with bulk states and shows less clear surface character. The lift of the spin degeneracy leads to radical change of the surface FS: (1) The radius of the FS hexagon is smaller by $30 \%$ compared to the nonrelativistic calculation; (2) in the $\bar{\Gamma} \bar{M}$ symmetry directions the hole lobes are formed. Another remarkable feature of the Bi(111) surface electronic structure is the very strong anisotropy of the spinorbit splitting: it is $\approx 0.2 \mathrm{eV}$ in the $\bar{\Gamma} \bar{M}$ direction and even more in the $\bar{\Gamma} \bar{K}$ direction.

The occurrence of strong spin-orbit splitting is confirmed when the calculations are compared to the experimental results. Figure 2 shows the calculated

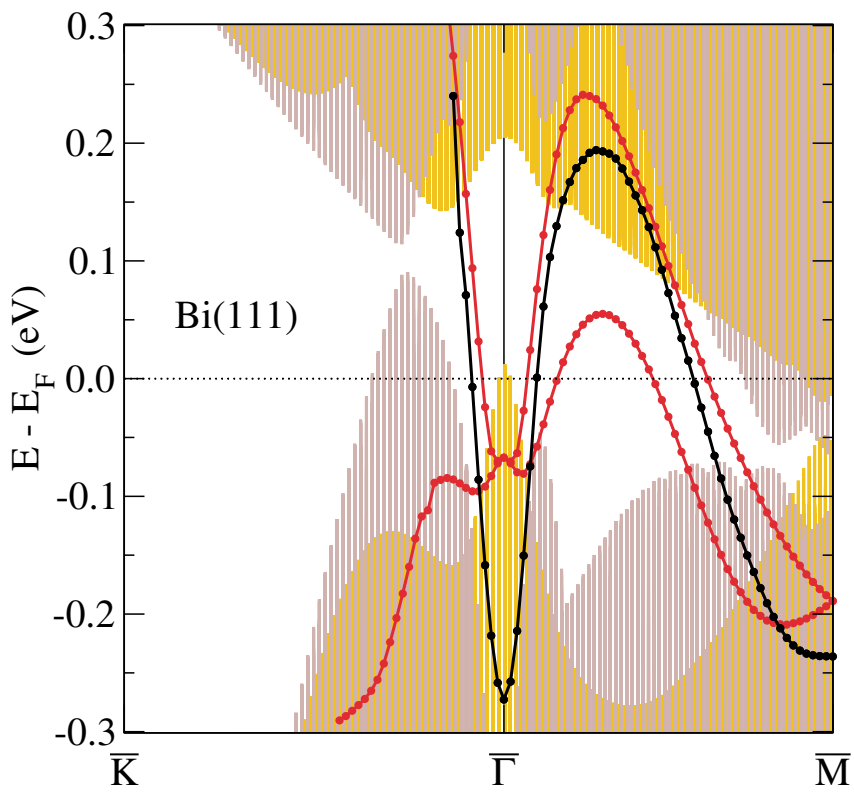

FIG. 1 (color). Surface states of $\mathrm{Bi}(111)$ calculated without (black) and with (red) spin-orbit splitting included. The shaded areas show the projection of the bulk bands obtained without (violet) and with (yellow) SOC and their superposition (brown). 
electronic structure for the three surfaces together with experimental data. In Fig. 2(a) we compare the results for $\mathrm{Bi}(111)$ and find excellent agreement for the two split surface states near $\bar{\Gamma}$. The experimental results also agree with recently published data for $\mathrm{Bi}(111)$ [10] but the two split bands appear better resolved here. Here, as in Ref. [10], the intensity of both surface states strongly decreases close to $\bar{\Gamma}$. This is most likely due to the overlap with the projected bulk band structure. The surface states are no longer genuine surface states but surface resonances, which penetrate much more deeply into the crystal and give a lower photoemission intensity. Although it is, therefore, not simple to decide if the states are, in fact, degenerate at $\bar{\Gamma}$ or not, we find no evidence for the latter. This discrepancy with the data of Refs. $[10,18]$ is most likely due to a sample misalignment, which later was found to have been present [11]. Our interpretation is in disagreement with the result from Ast and Höchst, who used a $\operatorname{Bi}(111)$ bilayer to simulate the surface-state dispersion of $\mathrm{Bi}(111)$ with a tight-binding model [19]. In their case, the calculated bands match the experimental dispersion beyond the $\bar{\Gamma}$ point when the bilayer thickness is increased by $70 \%$ with respect to the bulk value and when the SOC strength was reduced to $13 \%$ of the experimental value $1.5 \mathrm{eV}$ [13]. The bilayer, however, always has inversion symmetry, and therefore this calculation yields two spin-degenerate bands near the Fermi energy that do not cross at $\bar{\Gamma}$.

Figure 2(b) shows the situation near $\bar{\Gamma}$ of $\operatorname{Bi}(110)$. The experimental results have already been published elsewhere [7]. In the theory one can clearly see that, as in $\operatorname{Bi}(111)$, the surface state on $\operatorname{Bi}(110)$ is degenerate at $\bar{\Gamma}$ and splits into two surface states along the symmetry lines with one electron per $\mathbf{k}$ point. In contrast to $\mathrm{Bi}(111)$ this surface state is unoccupied at $\bar{\Gamma}$ and has negative effective electron masses that lead to the formation of the hole FS pocket around $\bar{\Gamma}$ [7]. This specific behavior of the surface- state bands is also responsible for the formation of the electron FS pocket between $\bar{X}_{1}$ and $\bar{M}_{1}$ and the hole pocket at $\bar{M}_{1}$ [7]. In the experiment, only the lower branch of the spin-orbit split state can be observed as it enters the occupied states. Such a situation can be highly confusing, because the band could be mistaken for a simple parabolic hole pocket.

The scenario of a very steep band and a flatter one near $\bar{\Gamma}$ can also be found on $\mathrm{Bi}(100)$. This is shown in Ref. [20] and is therefore not presented here. Instead, Fig. 2(c) shows the situation near the $\bar{M}^{\prime}$ point. This point is the $\bar{M}$ point of the quasihexagonal SBZ of $\mathrm{Bi}(100)$ which is not lying on the mirror plane of the SBZ (see Ref. [20]). Since all $\bar{M}$ points of a (quasi)hexagonal SBZ fulfill the criterion of lying exactly in the middle of the line joining two $\bar{\Gamma}$ points, we also expect a degenerate surface state here. As Fig. 2(c) shows, this is indeed the case. In fact, here the bands close to the high-symmetry point are so steep that the dispersion cannot be resolved in the experiment. In the rest of the SBZ, the agreement between experiment and calculation is more difficult to find. This is due to the deep penetration of the surface states into the bulk, such that even a calculation with a 22 layer film cannot completely avoid the interaction between the two surfaces. Details of the electronic structure of $\mathrm{Bi}(100)$ will be published elsewhere [21].

The spin-orbit splitting obtained for the Bi surfaces is a few times bigger than that of the surface state on $\mathrm{Au}(111)$ $[1,2]$, which is to be expected since the atomic spin-orbit splitting in $\mathrm{Bi}$ is 3 times larger than that in Au. However, the character of the spin-orbit splitting in the present case is different from that in $\mathrm{Au}(111)$. For the latter, the electronic structure can be described by a free electronlike two-dimensional surface state at $\bar{\Gamma}$. In such a case, the spin-orbit interaction can be treated by adding a socalled Rashba term to the nonrelativistic Hamiltonian [22]. This leads to a splitting of the surface state, which
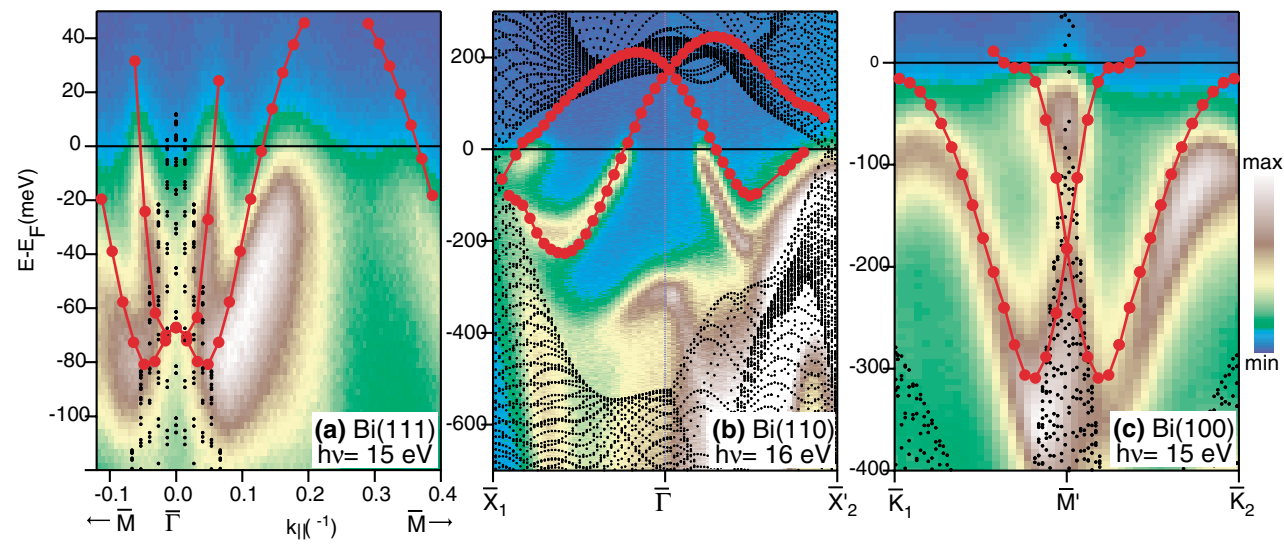

FIG. 2 (color). Calculated and measured electronic structure in the vicinity of two high symmetry points on three surfaces of Bi. (a) $\bar{\Gamma}$ on $\mathrm{Bi}(111)$, (b) $\bar{\Gamma}$ on $\mathrm{Bi}(110)$, and (c) $\bar{M}_{1}$ on $\mathrm{Bi}(100)$. The small black dots are the projected bulk band structure calculated using the tight-binding model of Liu and Allen [17]. The red solid circles are the calculated surface state energies; the thin red line is a guide to the eye. The photoemission intensity is linearly scaled from dark blue (minimum) to white (maximum). 
is linear in $\mathbf{k}$. In the case of $\mathrm{Bi}$, the surface states are not free electronlike and they are distributed over the whole SBZ; therefore, the spin-orbit splitting shows a much more complex behavior which can be revealed only by first-principle band structure methods.

The SOC-induced splitting should have some important consequences for the physical properties of the $\mathrm{Bi}$ surfaces, in particular, for the screening. In the Lindhard picture of screening, the susceptibility $\chi(\mathbf{q})$ is essentially given by an integral over all processes where an electron hops between an occupied state and an unoccupied state separated by $\mathbf{q}$. In a two-dimensional situation this type of screening can lead to a CDW-type instability only when there are "nested" elements of the FS, separated by $\mathbf{q}$. Such a situation exists for $\mathrm{Bi}(111)$, where the FS of the electron pocket around $\bar{\Gamma}$ is hexagonal [11]. Ast and Höchst have recently shown [11] that the leading edge of the energy distribution curves at the Fermi level crossing shifts discontinuously as a function of temperature, indicating the formation of a CDW. However, when we take into account the spin of the states involved in the alleged formation of the CDW, the electron hopping across the FS would have to undergo a spin flip because of the split nature of the bands. This makes the occurrence of a CDW very unlikely.

The spin-orbit splitting in surface bands on the Bi surfaces can also have drastic consequences for electron and hole dynamics in surface states. In particular, the surface response function should include all the spin-flip processes between the split surface bands with different spin direction. It can lead to the formation of surface spindensity waves even in cases when the nesting at the surface FS does not occur. The spin-orbit splitting should also lead to different hole (electron) lifetimes in surface states compared to that for the nonsplit surface state. This is due to both the surface response function that now includes spin-flip processes and to a different phase space factor $[9,23]$. The surface-state spin-orbit splitting can also affect the electron-phonon (e-ph) coupling on the Bi surfaces. Strong $e$-ph coupling in surface states on $\mathrm{Bi}(100)$ was already discussed by Gayone et al. [20], who assumed direct interaction between electron and phonon systems. Here we note that the existence of spin-split surface states also permits a spin-wave mediated $e$-ph interaction.

The lack of inversion symmetry and the large spinorbit splitting holds also at Bi-insulator interfaces, where spin-orbit split surface states turn to interface states. We speculate that ultrathin films of Bi covered with an insulator become much more effective spin filters and spin manipulators for injected spin-polarized electrons than semiconductors explored in spintronics because the relevant $k$ vectors and the difference of the $k$ vectors for spinup and spin-down electrons are much larger than for semiconductors.
In conclusion, we have presented the first ab initio calculation of the relativistic surface electronic structure for $\mathrm{Bi}(111), \mathrm{Bi}(110)$, and $\mathrm{Bi}(100)$. We have shown that the SOC term leads to a strong and anisotropic splitting of the surface-state bands that profoundly modifies the dispersion of the surface states and the surface FS. The calculated results are in good agreement with the experimental data if the spin-orbit splitting is taken into account. We have discussed the possible effect of the SOC interaction on the surface response function and new mechanisms of electron and hole decay in the Bi surface states in terms of electron-electron and electron-phonon interactions. We also discussed the possible use of this effect for spintronic applications.

This work was partially supported by the UPV/EHU, Spanish MCyT, the Max Planck Research Award Fund, and by the Danish National Science Foundation.

*Electronic addresses: philip@phys.au.dk http://www.phys.au.dk/ philip/

[1] S. LaShell, B. A. McDougall, and E. Jensen, Phys. Rev. Lett. 77, 3419 (1996).

[2] G. Nicolay et al., Phys. Rev. B 65, 033407 (2001).

[3] J. Henk, A. Ernst, and P. Bruno, Phys. Rev. B 68, 165416 (2003).

[4] E. Rotenberg, J.W. Chung, and S. D. Kevan, Phys. Rev. Lett. 82, 4066 (1999).

[5] M. Hochstrasser et al., Phys. Rev. Lett. 89, 216802 (2002).

[6] M. Hengsberger et al., Eur. Phys. J. B 17, 603 (2000).

[7] S. Agergaard et al., New J. Phys. 3, 15 (2001).

[8] E. Rotenberg, J. Schaefer, and S. D. Kevan, Phys. Rev. Lett. 84, 2925 (2000).

[9] P. M. Echenique et al., Chem. Phys. 251, 1 (2000).

[10] Ch. R. Ast and H. Höchst, Phys. Rev. Lett. 87, 177602 (2001).

[11] Ch. R. Ast and H. Höchst, Phys. Rev. Lett. 90, 016403 (2003).

[12] X. Gonze, J.-P. Michenaud, and J.-P. Vigneron, Phys. Rev. B 41, 11827 (1990).

[13] C. E. Moore, Atomic Energy Levels, Natl. Bur. Stand. (U.S.) Circular No. 467 (U.S. GPO, Washington, DC, 1949).

[14] E. Wimmer et al., Phys. Rev. B 24, 864 (1981); M. Weinert et al., ibid. 26, 4571 (1982).

[15] C. Li et al., Phys. Rev. B 42, 5433 (1990).

[16] S. V. Hoffmann et al., Nucl. Instrum. Methods Phys. Res., Sect. A 523, 441 (2004).

[17] Y. Liu and E. Allen, Phys. Rev. B 52, 1566 (1995).

[18] Ch. R. Ast and H. Höchst, Phys. Rev. B 66, 125103 (2002).

[19] Ch. R. Ast and H. Höchst, Phys. Rev. B 67, 113102 (2003).

[20] J. E. Gayone et al., Phys. Rev. Lett. 91, 127601 (2003).

[21] J. E. Gayone et al. (to be published).

[22] L. Petersen and P. Hedegård, Surf. Sci. 459, 49 (2000).

[23] J. Kliewer et al., Science 288, 1399 (2000). 